\title{
EDITORIAL
}

DDS - Die Deutsche Schule

113. Jahrgang 2021, Heft 3, S. 247-252

https://doi.org/10.31244/dds.2021.03.00

CC BY-NC-ND 4.0

Waxmann 2021

\section{Editorial zum Themenschwerpunkt: Kooperation von Eltern und Schule?!}

\section{Editorial to the Focus Topic: \\ Cooperation between Parents and Schools?!}

Verschiedenste empirische Befunde der Bildungsforschung der letzten Jahre verweisen auf die Bedeutung des familiären Hintergrundes für die Bildungsbiographien von Kindern und Jugendlichen. Dies gilt in besonderer Weise für Schüler*innen aus sozial benachteiligten Milieus, indem weniger erfolgreiche Bildungsbiographien über geringere familiäre Unterstützung und/oder habituelle Passungsprobleme erklärt werden (vgl. z. B. Chamakalayil, Ivanova-Chessex, Leutwyler \& Scharathow, 2021; Frank \& Sliwka, 2016). Gleichzeitig ist mit dem verpflichtenden Besuch der Schule die Hoffnung verknüpft, Bildungsungleichheiten, die durch ungleiche schulnahe familiäre Sozialisations- und Erziehungsprozesse entstehen, entgegenzuwirken.

Die persistierenden Befunde zur sozialen Selektivität des deutschen Schulsystems der letzten Jahrzehnte machen hingegen deutlich, dass dies nur bedingt gelingt. Knüpft man an die aufgeworfenen Befunde zur Bedeutung der Familie als Sozialisations-, Erziehungs-, und Bildungsinstanz an, so erscheint es folgerichtig, einen Fokus auf Möglichkeiten zur Optimierung der Zusammenarbeit von Erziehungsberechtigten und Schule zu richten. Entsprechend verweist der bildungswissenschaftliche Diskurs seit Jahrzehnten auf die positive Wirkung, die eine Zusammenarbeit von Schule und Eltern etwa auf die Lernentwicklung oder auch die Lernmotivation entfalten kann (z. B. Krumm, 1988; Sacher, 2014).

Was sich jedoch hinter der Zusammenarbeit von Eltern und Schule verbirgt, erscheint durchaus inhaltlich divers und nicht zuletzt abhängig von der Gestalt des Schulsystems und/oder gesamtgesellschaftlichen Entwicklungen. So ist anzunehmen, dass der häuslichen Unterstützung beim Lernen im Rahmen eines Halbtagsschulsystems eine höhere Bedeutung zukommt als in Ganztagsschulsystemen. Darüber hinaus gibt es unterschiedliche Möglichkeiten, aber auch Pflichten der Elternbeteiligung, die ge- 
sellschaftlich tradiert und/oder schulgesetzlich (inter)national unterschiedlich verankert sind. Mit Blick auf Erziehungsberechtigte aus benachteiligten Milieus stellt sich die Frage, wie Zusammenarbeit von Eltern und Schule ohne einseitige Macht und Deutungshoheiten prozessiert werden kann und wechselseitige Anerkennungsprozesse ermöglicht werden können (vgl. Ivanova-Chessex, 2020). Nicht zuletzt scheint es Grenzen gewünschter Elternmitwirkungen zu geben, denkt man etwa an Schilder vor Grundschultoren, auf denen steht: „Ab hier schaffe ich es allein. Eltern bleiben bitte draußen“.

Diese hier sichtbar werdende zeitliche und räumliche Trennung von Familie und Schule erfährt in der aktuellen Zeit des pandemiebedingten Distanzlernens eine temporäre Auflösung. Entsprechend rückt die Bedeutung der elterlichen Unterstützung durch vorhandene oder weniger vorhandene personale, fachliche sowie sächliche Ressourcen in den Fokus und wird aktuell insbesondere mit Blick auf mögliche Folgen der Verschärfung von Bildungsungleichheit diskutiert.

Kooperation von Eltern und Schule scheint entsprechend - je nach Akteursperspektive oder räumlich-zeitlicher Verortung - durchaus ambivalent normativ aufgeladen und unterschiedlich definitorisch konzeptualisiert zu werden, wie an Begriffen wie „Erziehungs- und Bildungspartnerschaften“, „Elternarbeit“, „Elternpartizipation“ oder „Elternbildung" etc. deutlich wird. Entsprechend der unterschiedlichen Konzepte sind unterschiedliche Wirkungen auf die Bildungsbiographie zu erwarten - wobei auch Wirkungen unterschiedlich moderiert sein können und nicht allein auf fachliches Lernen fokussiert sein sollten.

Im Rahmen dieses Heftes knüpfen wir nun an die vielfältigen Anschlussmöglichkeiten dieses Themenfeldes an.

Im Sinne einer Einführung führt der Beitrag von Dagmar Killus und Angelika Paseka zum Heftthema hin und gibt eine Orientierung im Feld. Dabei fokussieren die Autorinnen zunächst auf die Voraussetzungen für die Kooperation von Eltern und Schule und betrachten neben rechtlichen Rahmenbedingungen auch verschiedene Adressierungen von Eltern durch Lehrpersonen, Schule und Schulverwaltung. Anschließend werden Begriffe und Diskurse betrachtet, wobei zwischen einem eher normativen und einem eher wissenschaftlichen Diskurs unterschieden wird. Darauf folgend werden fünf Forschungsstränge vorgestellt, mit denen sich die aktuelle Forschungslage systematisieren lässt. Mit einem aktuellen Bezug werden in dem Beitrag zudem empirische Befunde zur Kooperation unter Pandemiebedingungen betrachtet. Der Beitrag schließt mit einem Fazit, in dem unter anderem zukünftige Entwicklungsbereiche in der Kooperation von Eltern und Schule skizziert werden.

In den folgenden zwei Schwerpunktbeiträgen wird Bezug auf aktuelle empirische Studien zur Kooperation von Eltern und Schule genommen: 
Judith Adamczyk fokussiert das Vertrauen von Eltern in die Schule, das eine wesentliche Voraussetzung für das Funktionieren von Schule darstellt und angesichts der pandemiebedingten Situation derzeit eine besondere Bedeutung besitzt. Vor dem Hintergrund eines spärlichen Forschungsstandes zur Thematik präsentiert die Autorin eine explorative Studie, in deren Rahmen Eltern als Akteur ${ }^{\star}$ innen - samt ihren Erwartungen, Wissensbeständen und emotionalen Bewertungen von Schule - vertrauenstheoretisch in den Blick genommen werden. Auf der Basis von qualitativen Interviews mit Eltern schulpflichtiger Kinder unterschiedlichen Bildungshintergrunds zeigt sie mittels einer kategorienbasierten Auswertung, dass die Mehrheit der Eltern ein positives Vertrauensverhältnis mit der Schule aufweist, und dass nahezu alle Eltern auf einer eher kind- bzw. lernbezogenen Ebene (z. B. Hilfe bei Hausaufgaben) sowie auf der Ebene der Schule (Besuche von Elternabenden) an Schule beteiligt sind. Anhand einer typenbildenden Inhaltsanalyse unter Einbeziehung der Kategorien „Höhe des Vertrauens" und „Beteiligung an Schule“ generiert sie zudem vier kontrastreiche Elterntypen. Die Autorin stellt in ihrem Beitrag abschließend die Verantwortung der Schule bei der Stärkung des Vertrauens von Eltern (sowie Schüler*innen) während der derzeitigen pandemischen Situation heraus, die für den Aufbau positiver Beziehungen und einen daraus resultierenden positiven Einfluss auf den Bildungserfolg der Kinder und die Zufriedenheit der Eltern erforderlich sei.

Sabrina Bonanati, Nicole Gruchel, Ricarda Kurock und Heike M. Buhl beschäftigen sich in ihrem Beitrag mit den Langzeiteffekten des Family-Literacy-Programms „LIFE - Lesen in Familie erleben" auf die elterliche Unterstützung beim Lesenlernen. Bei LIFE handelt es sich um ein standardisiertes schulbasiertes Programm, das in sechs Veranstaltungen über das erste Schuljahr hinweg stattfindet und das den Fokus auf die Förderung spielerischer Interaktionen bei der Auseinandersetzung mit Texten legt. Die Autorinnen setzen damit an einer Forschungslücke an, denn zwar gebe es bereits verschiedene Materialien und Konzepte, um Family-Literacy-Programme in Schulen zu implementieren, systematische Studien, die sich mit der Wirksamkeit solcher Programme beschäftigen, fehlten jedoch. Die empirische Untersuchung der Effekte des Programms LIFE basiert auf einem quasi-experimentellen Design mit Experimentalund Kontrollgruppe. Neben der Elternperspektive wird auch die Wahrnehmung der Kinder auf die elterliche Unterstützung betrachtet. Die Autorinnen kommen zu dem Ergebnis, dass LIFE sowohl direkt nach dem Programm als auch ein halbes Jahr später positiv auf verschiedene Arten der Anschlusskommunikation beim gemeinsamen Lesen wirkte. Die Ergebnisse werden u. a. mit Fokus auf die Herausforderungen, die mit der Durchführung von Family-Literacy-Programmen einhergehen, diskutiert.

In fünf Berichten wird die Thematik in ihren unterschiedlichen Facetten weiter entfaltet.

Janina Täschner, Doris Holzberger und Delia Hillmayr gehen in ihrem Forschungsbericht der Frage nach, inwiefern verschiedene Formen der Elternbeteiligung die 
schulische Leistung und Motivation von Schülerinnen und Schülern fördern können. Vor dem Hintergrund einer Unterscheidung der Beteiligungsdimensionen (1) homebased parental involvement als Verhaltensweisen im Kontext der Gestaltung von Lerngelegenheiten im familiären Umfeld, (2) school-based parental involvement als Verhaltensweisen im Raum Schule und (3) academic socialization als Verhaltensweisen, die auf die Kommunikation bildungsrelevanter Inhalte bezogen sind, führen sie erstmals eine nach Dimensionen differenzierende Zusammenhangsanalyse durch. Mittels einer systematischen Zusammenfassung von 18 vorliegenden Überblicksarbeiten zur Thematik (Second-Order-Review) identifizieren sie größtenteils positive, aber je nach Dimension unterschiedlich große Effekte. Aus ihren Befunden leiten die Autorinnen verschiedene Möglichkeiten der gezielten schulischen Unterstützung von Elternbeteiligung ab.

Julia Hugo befasst sich mit der Kooperation von Eltern und Schule aus einer rechtlichen Perspektive und stößt damit in eine im bildungswissenschaftlichen Diskurs eklatante Lücke. Sie betrachtet die Rolle von Eltern im Schulverhältnis als bestehendem Rechtsverhältnis und nimmt dabei zunächst einen historischen Blick ein, indem sie auf den Gesetzesvorbehalt im Schulverhältnis eingeht, durch den seit den 1970er Jahren z.B. jeder wesentliche Eingriff in die Rechte von Kindern, aber auch von Eltern, im Schulverhältnis als öffentlichem Rechtsverhältnis gesetzlich zu regeln ist. Sodann betrachtet sie die aktuelle Situation anhand der Schulgesetze der Länder, wobei sie den Fokus exemplarisch auf Bayern legt. Schließlich thematisiert sie Grenzen elterlicher Rechte.

Jennifer Adolé Akue-Dovi, Imogen Feld, Julia Gerick, Hannah van Riel und Eric Vaccaro betrachten das Hamburger Projekt "SchulMentoren - Hand in Hand für starke Schulen“, in dem das Elternmentoring eine zentrale Projektkomponente darstellt, aus der Perspektive der Bildungsforschung und der Bildungsadministration. In ihrem Bericht wird auf Grundlage der Ergebnisse der wissenschaftlichen Projektevaluation der Frage nachgegangen, wie Barrieren zwischen Schule und Elternhaus abgebaut werden können. Dazu werden sowohl quantitative als auch qualitative Daten herangezogen. Abschließend werden mögliche Implikationen und zukünftige Perspektiven expliziert.

Nils Hartung, Susanne Geller, Nils Euker und Alexandra Marx stellen das Konzept der Preisträgerschule Mosaikschule Marburg des Deutschen Schulpreises vor. Die Mosaikschule Marburg ist eine selbstständige Förderschule, die den Anspruch verfolgt, Schülerinnen anknüpfend an ihre Stärken und Bedürfnisse zu gesellschaftlicher Teilhabe zu befähigen. Die Zusammenarbeit mit den Erziehungsberechtigten stellt eine wesentliche Basis zur Realisierung individueller Lernangebote dar und wird in verschiedener Weise kontinuierlich und systematisch verfolgt, was im Rahmen des Berichtes vertiefend - insbesondere auch mit Blick auf die Zeit der Pandemie - betrachtet wird. 
Sabine Walper stellt anknüpfend an wesentliche Ergebnisse des Neunten Familienberichts zentrale Aspekte der aktuellen Situation von Familien in Deutschland vor. So verweist sie etwa auf die gestiegenen Ansprüche an Elternschaft, die sie im Kontext einer zunehmenden Diversität von Familien insgesamt und mit Blick auf soziale Ungleichheit als besondere gesellschaftliche Herausforderung verortet und diskutiert. Um eine Verschärfung sozialer Ungleichheit zu vermeiden, sollte die Zusammenarbeit von Schule und Eltern im Sinne einer Stärkung von Erziehungs- und Bildungspartnerschaften verbessert werden. Hierzu ist es jedoch nötig, die systemischen, organisationalen Bedingungen und kompetenzbezogenen Voraussetzungen zu schaffen, etwa indem entsprechende Zeitkontingente der Lehrkräfte standortspezifisch zu realisieren wären. Darauf aufbauend begründet sie ein Konzept für Schule als Bildungsort nicht nur für Schüler*innen, sondern auch für Eltern, das sie mit Blick auf unterschiedliche Facetten erörtert.

Dieses Heft enthält außerhalb des Themenschwerpunkts einen weiteren Beitrag zur Diskussion. Zur Kompensation antizipierter Lernrückstände von Schüler*innen aufgrund der Schulschließungen hat die Bundesregierung ein Aktionsprogramm mit einem Volumen von zwei Milliarden Euro aufgelegt. Detlef Fickermann und Ilka Hoffmann diskutieren in ihrem Beitrag Modi der Mittelverteilung und deren Konsequenzen insbesondere mit der Perspektive der Förderung benachteiligter Schüler ${ }^{\star}$ innen. Ausgangspunkt der Diskussion bildet eine kritische Analyse des sogenannten Königsteiner Schlüssels, der in der Vergangenheit zur Mittelverteilung eingesetzt wurde. Die Autor*innen plädieren auf der Grundlage hier präsentierter, differenzierter Berechnungen dafür, forschungsgestützte Empfehlungen zur Auswahl des Verteilungsschlüssels zugrunde zu legen, so dass etwa die unterschiedlichen Ausgangslagen der Länder Berücksichtigung finden.

Kathrin Dedering, Julia Gerick \& Kathrin Racherbäumer

\section{Literatur und Internetquellen}

Chamakalayil, L., Ivanova-Chessex, O., Leutwyler, B., \& Scharathow, W. (2021). „.. wieder das Klassen-Ding“: Bildung als biographischer Positionierungsprozess in familialen Verwobenheiten. In A. Schondelmayer, C. Riegel \& S. Fitz-Klausner (Hrsg.), Familie und Normalität: Diskurse, Praxen und Aushandlungsprozesse (S. 61-76). Opladen: Budrich. https://doi.org/10.2307/j.ctvlbvndpc.6

Frank, S., \& Sliwka, A. (2016). Eltern und Schule: Aspekte von Chancengerechtigkeit und Teilhabe an Bildung. Weinheim \& Basel: Beltz Juventa.

Ivanova-Chessex, O. (2020). Verhältnisbestimmungen zwischen Eltern und Schule im Kontext hegemonialer Normen und Ungleichheitsverhältnisse: Stand und Perspektiven der Forschung. Zeitschrift für Soziologie der Erziehung und Sozialisation, 40 (2), 172187.

Krumm, V. (1996). Über die Vernachlässigung der Eltern durch Lehrer und Erziehungswissenschaft. Plädoyer für eine veränderte Rolle der Lehrer bei der Erziehung 
| Editorial

der Kinder. In A. Leschinsky (Hrsg.), Die Institutionalisierung von Lehren und Lernen. Beiträge zu einer Theorie der Schule (Zeitschrift für Pädagogik, 34. Beiheft) (S. 119137). Weinheim u. a.: Beltz.

Sacher, W. (2014). Elternarbeit als Erziehungs- und Bildungspartnerschaft. Grundlagen und Gestaltungsvorschläge für alle Schularten. Bad Heilbrunn: Klinkhardt. 